\title{
Repopulation of the Vg2Vd2 Repertoire After Prolonged HAART Nadia Propp* ${ }^{* \ddagger}$, Cristiana Cairo, Andrew Hebbeler and C David Pauza
}

Address: Institute of Human Virology, Baltimore, MD, 212101

* Corresponding author $\ddagger$ Presenting author

from 2005 International Meeting of The Institute of Human Virology Baltimore, USA, 29 August - 2 September 2005

Published: 8 December 2005

Retrovirology 2005, 2(Suppl I):PI55 doi:I0.I I86/I742-4690-2-SI-PI55

HIV infection leads to a rapid and complete loss of cells expressing the Vg2-Jg1.2 chain of the gd T cell receptor. This cell subset is generally measured by spectratyping and is defined as $\operatorname{Vg} 2$ chains encoded by sequences between 990 and 996 nucleotides in length. In a previous cross-sectional study (Bordon, et al. JID 189:1482, 2004) we demonstrated a relationship between the duration of therapy and recovery of Vg2 sequences between 990-996 nucleotides. Now using longitudinal specimens and extensive analysis of $\mathrm{Vg} 2$ chains by DNA sequencing, we show that prolonged HAART does indeed reconstitute the repertoire of 990-996 length Vg2 chains, but they are no longer restricted to using the Jg1.2 segment. Instead, we observe Jg1.1 and Jg2.3 sequences with unusual $\mathrm{N}$ regions sequences that are rare in healthy individuals. In this unique example, we show that HIV infection exhausts the population of $\mathrm{T}$ cell clones capable of expressing the Vg2Jg1.2 chain, but that strong selection for $\mathrm{Vg} 2$ chains in the range of 990-996 nucleotides, forces the expansion of a previously minor population. Within the $\mathrm{Vg} 2 \mathrm{Vd} 2 \mathrm{~T}$ cell population, prolonged therapy enabled a functional reconstitution of the Vg2 repertoire. Even though repertoire selection for $\mathrm{Vg} 2$ is largely independent of thymic function, there was no evidence that $\mathrm{T}$ cell clones could be replaced from hematopoietic stem cells, despite the continued strong selection for $\mathrm{Vg} 2$ chains of the appropriate length. 